similar HR: 1.39 [0.71-2.74] in patients with PD-L1 IC $<5 \%$ (mPFS 19.3mo [14.9-25.7] vs mPFS $18.2 \mathrm{mo}$ [14.5-19.3] in control and experimental arms respectively). Pembrolizumab improved mPFS (HR: 0.56 [0.19-1.61]) for pts with PD$\mathrm{L} 1 \geq 5 \%$ (mPFS 20.8 mo [9.5-NE] vs mPFS $23.4 \mathrm{mo}$ [18.0-NE] in control and experimental arms respectively).

Conclusion* If no benefit in adding Pembrolizumab to CT +/bevacizumab was found in wtBRCA subgroup, exploratory PFS analyses in the PD-L1 IC $\geq 5 \%$ subgroup showed a trend favouring Pembrolizumab in patients with advanced HGSOC.

\section{PROGNOSTIC IMAGE-BASED QUANTIFICATION OF CD8CD103 T CELL SUBSETS IN HIGH-GRADE SEROUS OVARIAN CANCER PATIENTS}

\begin{abstract}
${ }^{1,6}$ ST Paijens, ${ }^{1,6} \mathrm{~A}$ Vledder* ${ }^{*}, 6 \mathrm{D}$ Loiero, ${ }^{3} \mathrm{EW}$ Duiker, ${ }^{3} \mathrm{~J}$ Bart, ${ }^{4} \mathrm{AM}$ Hendriks, ${ }^{4} \mathrm{M}$ Jalving, ${ }^{1} \mathrm{HH}$ Workel, ${ }^{3} \mathrm{H}$ Hollema, ${ }^{3} \mathrm{~N}$ Werner, A Plat ${ }^{1},{ }^{1} \mathrm{GBA}$ Wisman, ${ }^{1} \mathrm{R}$ Yigit, ${ }^{1} \mathrm{H}$ Arts, ${ }^{5} \mathrm{AJ}$ Kruse, ${ }^{5} \mathrm{NM}$ de Lange, ${ }^{2,7} \mathrm{VH}$ Koelzer, ${ }^{1,7} \mathrm{M}$ de Bruyn, ${ }^{1,7} \mathrm{HW}$ Nijman. ${ }^{1}$ University of Groningen, University Medical Center Groningen, Department of Obstetrics and Gynecology, The Netherlands; ${ }^{2}$ Department of Pathology and Molecular Pathology, University Hospital and University of Zurich, Switzerland; ${ }^{3}$ University of Groningen, University Medical Center Groningen, Department of Pathology, The Netherlands; ${ }^{4}$ University of Groningen, University Medical Center Groningen, Department of Medical Oncology, The Netherlands; ${ }^{5}$ /sala Hospital Zwolle, Department of Obstetrics and Gynecology, The Netherlands; ${ }^{6}$ Contributed equally; ${ }^{7}$ Shared senior authorship
\end{abstract}

10.1136/ijgc-2021-ESGO.370

Introduction/Background* CD103-positive tissue resident memory-like CD8 + T cells (CD8CD103 TRM) are associated with improved prognosis across malignancies, including highgrade serous ovarian cancer (HGSOC). We investigated whether quantification of CD8, CD103 or both is required to improve existing survival prediction and whether all HGSOC patients or only specific subgroups of patients benefit from infiltration.

Methodology We applied image-based quantification of CD8 and CD103 multiplex immunohistochemistry in the intratumoral and stromal compartments of 268 advanced-stage HGSOC patients from two independent clinical institutions.

Result(s)* Infiltration denisty of CD8CD103 TRM was independent of clinicopathological factors and primary treatment strategy. A survival benefit of CD8CD103 TRM infiltration was observed in patients treated with primary cytoreductive surgery. Moreover, survival benefit in this group was limited to patients with no macroscopic tumor lesions after surgery (high epithelial CD8CD103 TRM infiltration 5 year survival $83 \%$ versus $52 \%, \mathrm{p}=0.03$; high stromal CD8CD103 TRM 5 year survival $77 \%$ versus $54 \%, p=0.01)$. No effect of CD8CD103 TRM infiltration on overall survival was observed in patients treated with neo-adjuvant chemotherapy, with or without macroscopic tumor lesions after surgery (high epithelial CD8CD103 TRM infiltration, $p=0.77$; high stromal CD8CD103 TRM infiltration, $p=0.32$ ).

Conclusion* Our results suggest CD8CD103 TRM quantification as a superior method for prognostication compared to single CD8 or CD103 quantification, and supports the further exploration of image-based quantification of CD8CD103 TRM in HGSOC. This approach provides novel insights into

a. High CD8CD103 TRM stromal and epithelial infiltration, HGSOC tissue

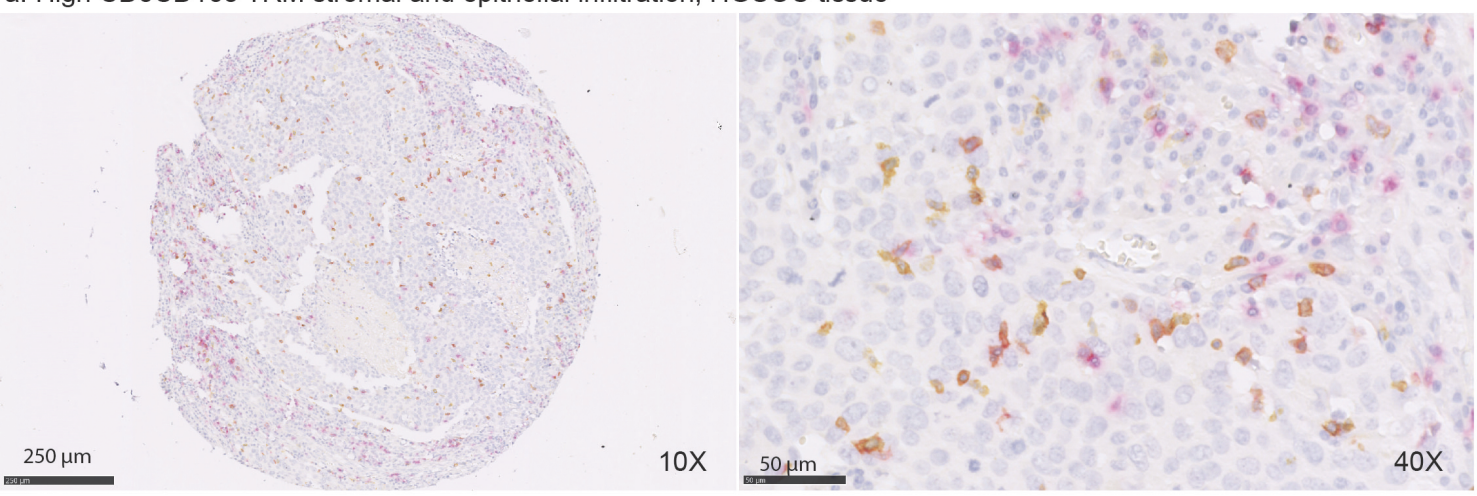

b. Low CD8CD103 TRM stromal and epithelial infiltration, HGSOC tissue

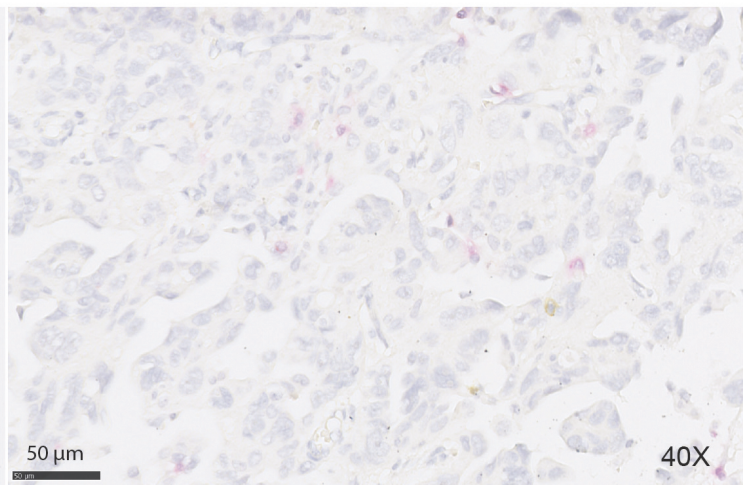

Abstract 263 Figure 1 Examples of representation CD8CD103 double staining assessed by immunohistochemistry 
a. Overall Survial PDS cohort

CD8CD103 TRM tumor epithelial infiltration
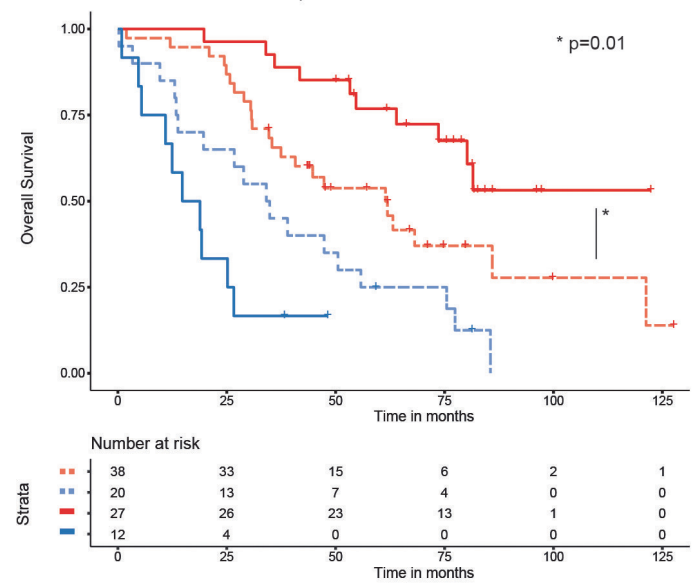

c. Overall Survival NACT cohort

CD8CD103 TRM tumor epithelial infiltration
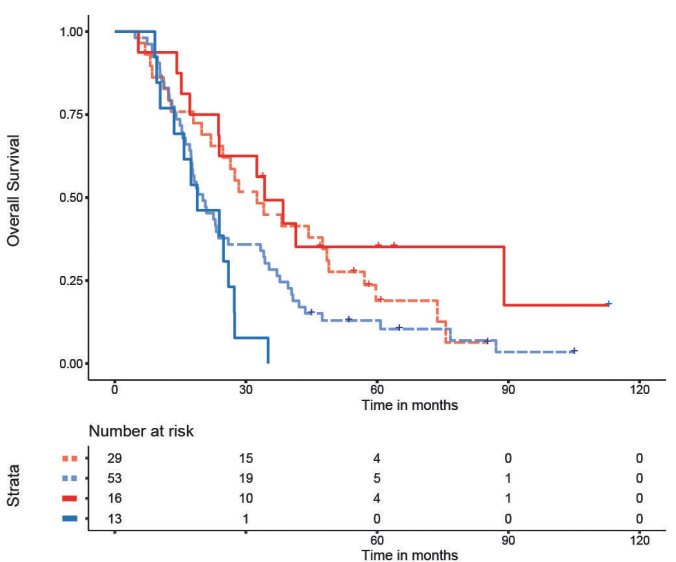

1=1= Low CD8CD103 infiltration + No macroscopic leasions post-surgery

High CD8CD103 infiltration + No macroscopic leasions post-surgery

=-1= Low CD8CD103 infiltration + Macroscopic leasions post-surgery

High CD8CD103 infiltration + Macroscop leasions post-surgery b. Overall Survival PDS cohort

CD8CD103 TRM Stromal infiltration
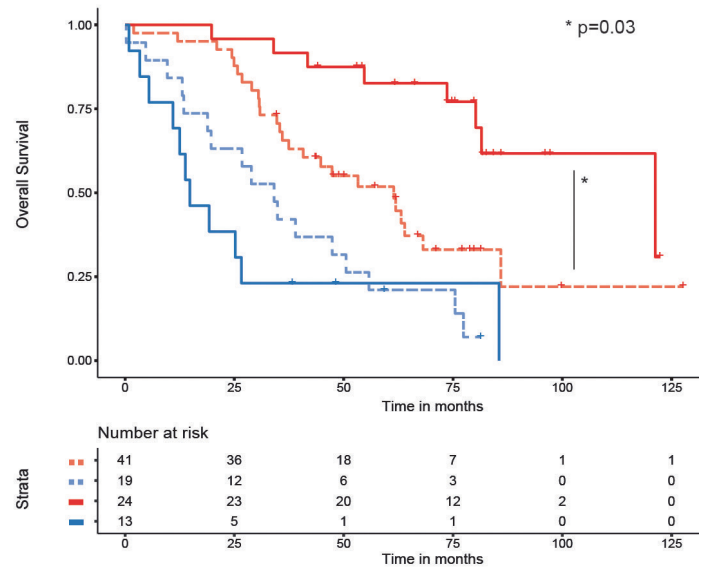

d. Overall Survival NACT cohort

CD8CD103 TRM Stromal infiltration
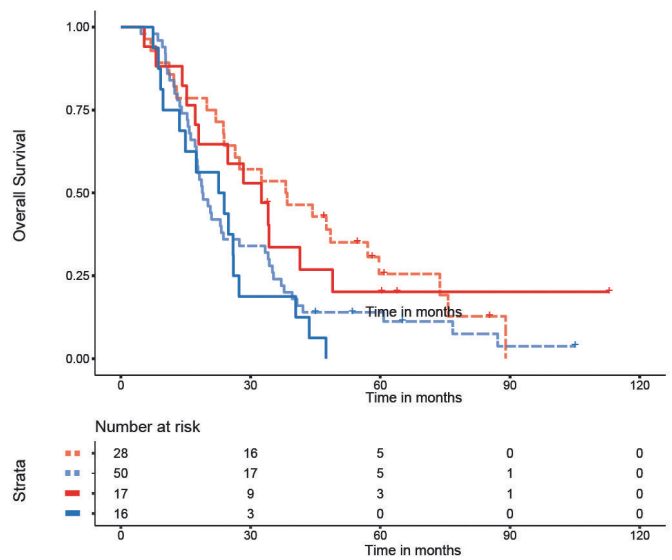

Abstract 263 Figure 2 Prognostic benefit of stomal and epithellial CD8CD103 TRM infiltration in patient subgroups

prognostic stratification of HGSOC patients and may contribute to personalized treatment strategies in the future.

\section{BIOMARKER-BASED EARLY DETECTION OF EPITHELIAL OVARIAN CANCER BASED ON A FIVE-PROTEIN SIGNATURE IN PATIENT'S PLASMA - A PROSPECTIVE TRIAL}

${ }^{1} \mathrm{D}$ Eichkorn* ${ }^{*}{ }^{2} \mathrm{~A}$ Hasenburg, ${ }^{3} \mathrm{~F}$ Vosshagen, ${ }^{4} \mathrm{E}$ Obermayr, ${ }^{5} \mathrm{~A}$ Geroldinger, ${ }^{6} \mathrm{R}$ Zeillinger, ${ }^{7} \mathrm{M}$ Bossart. ${ }^{1}$ Schwarzwald-Baar Clinics, Department of Obstetrics and Gynecology, Villingen-Schwenningen, Germany; ${ }^{2}$ University Medical Center Mainz, Department of Obstetrics and Gynecology, Mainz, Germany; ${ }^{3}$ Ortenau Clinics, Department of Anesthesiology, Lahr/Schwarzwald, Germany; ${ }^{4}$ Medical University of Vienna, Department of Obstetrics and Gynecology, Wien, Austria; ${ }^{5}$ Medical University of Vienna, Section for Clinical Biometrics, Center for Medical Statistics, Wien, Austria; ${ }^{6}$ Medical University of Vienna, Department of Obstetrics and Gynecology, Vienna, Austria; 'St. Josefskrankenhaus, Freiburg, Obstetrics, Gynecology and Gynecologic Oncology, Freiburg im Breisgau, Germany

10.1136/ijgc-2021-ESG0.371

Introduction/Background* Trial on five plasma biomarkers (CA125 - cancer antigen 125, HE4 - human epididymis protein 4, OPN - osteopontin, leptin, prolactin) and their possible role in differentiating benign from malignant ovarian tumors.

Methodology In this unicentric prospective trial preoperative blood samples of 43 women with ovarian masses determined for ovarian surgery were analyzed. 25 patients had pathologically confirmed benign, 18 malignant ovarian tumors. Blood plasma was analyzed for CA125, HE4, OPN, leptin, prolactin and MIF (macrophage migration inhibitory factor) by multiplex immunoassay analysis. Each single protein and a logistic regression model including all the listed proteins were tested as preoperative predictive marker for suspect ovarian masses.

Result(s)* Plasma CA125 was confirmed as a highly accurate tumor marker in ovarian cancer. HE4, OPN, leptin and prolactin plasma levels differed significantly between benign and malignant ovarian masses. With a logistic regression model a formula including CA125, HE4, OPN, leptin and prolactin was developed to predict malignant ovarian tumors. With a discriminatory AUC (area under the curve) of 0.96 it showed to be a highly sensitive and specific diagnostic test for a malignant ovarian tumor. 